\title{
Insight into Disease Processes through Studies of the Intracellular Vesicle Transport System
}

\section{Noah Isakov*}

The Shraga Segal Department of Microbiology, Immunology and Genetics, Faculty of Health Sciences and the Cancer Research Center, Ben Gurion University of the Negev, Beer Sheva, Israel

Eukaryotic cells synthesize different types of molecules that are transported within the cell to discrete areas, or secreted outside of the cell, in small packages, called vesicles. The mechanism by which intracellular vesicles carrying distinct cargo molecules are delivered to specific cellular compartments, and the means by which vesicles fuse with specific organelles or the plasma membrane, to deliver their cargo, is the focus of intense investigation by many laboratories. Studies performed by three pioneering scientists, who won the 2013 Nobel Prize in Physiology or Medicine, contributed significantly to the understanding of these mechanisms. The three Laureates, James E. Rothman, from the Department of Cell Biology, at Yale University in New Haven, Connecticut, Randy W. Schekman, from the Department of Molecular and Cell biology, at the University of California at Berkeley, California, and Thomas C. Südhof, from the Department of Molecular and Cellular Physiology at Stanford University, in Stanford, California, were jointly awarded the Nobel Prize "for their discoveries of machinery regulating vesicle traffic, a major transport system in our cells".

Randy W. Schekman utilized the baker's yeast, Saccharomyces cerevisiae, to study vesicle transport and fusion [1], and by screening a large number of temperature-sensitive mutants, he succeeded in identifying 23 genes that participate in the regulation of intracellular transport [2]. Moreover, based on the accumulation of membranes reflecting blocks in the traffic from the endoplasmic reticulum (ER), Golgiapparatus, or cell surface, he was able to identify genes that are involved in vesicle transport to distinct cellular organelles, or to the plasma membrane [3]. The work by Schekman provided a genetic basis for vesicle traffic and fusion by identifying key proteins that are essential for the vesicle transport system.

James E. Rothman used a biochemical in vitro reconstitution approach to identify molecules that participate in intracellular vesicle fusion processes. He discovered several new proteins that are essential for vesicle fusion with appropriate target membranes [4,5], and demonstrated the critical role of the SNARE proteins in vesicle delivery to specific subcellular compartments [6,7]. By showing that particular SNARE proteins in a vesicle ( $\mathrm{v}$-SNARE) interact with just one or few of the many types of potential SNARE proteins in the target vesicle ( $t$-SNARE), Rothman provided evidence for the directionality of the transport mechanism, which enables vesicles deliver their cargo to specific destinations within the cell.

Thomas C. Südh of studied the processes by which nerve cells communicate with each other, focusing on those that regulate the rapid exocytosis of synaptic vesicles and the release of neurotransmitters. He elucidated the mechanism by which calcium ions trigger the release of neurotransmitters and discovered and characterized proteins that are essential for the calcium-regulated vesicle fusion machinery $[8,9]$. Studies by Südhof unraveled the mechanisms by which intracellular calcium signals are being sensed and translated into the transport and fusion of vesicles. His important discoveries shed light on the mechanisms that regulate the temporal and precise control of vesicle fusion.

The discoveries made by the three new Nobel Laureates represent a major contribution to our understanding of the spatial and temporal events that enable the delivery of specific cargo molecules, with timing and precision, to precise destinations within and outside of cells. Furthermore, these studies provide mechanistic explanations to pathological conditions whereby disturbances in the vesicle transport mechanisms result in specific diseases, including neurological and immunological disorders, as well diseases of the endocrine system, such as diabetes.

\section{References}

1. Novick P, Schekman R (1979) Secretion and cell-surface growth are blocked in a temperature-sensitive mutant of Saccharomyces cerevisiae. Proc Natl Acad Sci U S A 76: 1858-1862.

2. Novick P, Field C, Schekman R (1980) Identification of 23 complementation groups required for post-translational events in the yeast secretory pathway. Cell 21: 205-215

3. Schekman R, Orci L (1996) Coat proteins and vesicle budding. Science 271 1526-1533.

4. Malhotra V, Orci L, Glick BS, Block MR, Rothman JE (1988) Role of an $\mathrm{N}$-ethylmaleimide-sensitive transport component in promoting fusion of transport vesicles with cisternae of the Golgi stack. Cell 54: 221-227.

5. Clary DO, Griff IC, Rothman JE (1990) SNAPs, a family of NSF attachment proteins involved in intracellular membrane fusion in animals and yeast. Cell 61: 709-721.

6. Weber T, Zemelman BV, McNew JA, Westermann B, Gmachl M, et al. (1998) SNAREpins: minimal machinery for membrane fusion. Cell 92: 759-772.

7. McNew JA, Parlati F, Fukuda R, Johnston RJ, Paz K et al. (2000) Compartmental specificity of cellular membrane fusion encoded in SNARE proteins. Nature 407: 153-159.

8. McMahon H T, Missler M, Li C, Sudhof T C (1995) Complexins: cytosolic proteins that regulate SNAP receptor function. Cell 83: 111-119.

9. Perin M S, Fried V A, Mignery G A, Jahn R, Sudhof T C (1990) Phospholipid binding by a synaptic vesicle protein homologous to the regulatory region of protein kinase C. Nature 345: 260-263.

*Corresponding author: Noah Isakov, The Shraga Segal Department of Microbiology, Immunology and Genetics, Faculty of Health Sciences, Ben Gurion University of the Negev, P.O.B. 653, Beer Sheva 84105, Israel, Tel: 972-8-6477267; Fax: 972-8-6477626; E-mail: noah@bgu.ac.il

Received December 25, 2013; Accepted December 30, 2013; Published January 01,2014

Citation: Isakov N (2014) Insight into Disease Processes through Studies of the Intracellular Vesicle Transport System. J Clin Case Rep 4: e125 doi:10.4172/21657920.1000 e125

Copyright: (C) 2014 Isakov N. This is an open-access article distributed under the terms of the Creative Commons Attribution License, which permits unrestricted use, distribution, and reproduction in any medium, provided the original author and source are credited. 